\title{
SEMISYNTHETIC COUMERMYCINS. I
}

\section{PREPARATION OF 3-ACYLAMIDO-4-HYDROXY-8-METHYL-7-[3-O- (5-METHYL-2-PYRROLYLCARBONYL)NOVIOSYLOXY]COUMARINS*}

\author{
J. G. Keil, I. R. Hooper, M. J. Gron, O. B. Fardig, \\ D. E. Nettleton, F. A. O'Herron, E. A. Ragan, \\ M. A. Rousche, H. Schmitz, R. H. Schreiber \\ and J. G. GODFREY
}
Department of Biochemical Research, Bristol Laboratories, Division of Bristol-Myers Company, Syracuse, New York, U.S. A.

(Received for publication August 29, 1968)

The ditetrahydropyranyl ether of coumermycin $A_{1}$ was prepared and converted to a series of new derivatives by an acyl interchange reaction. The mechanism of this interesting and useful reaction is discussed. All of the 67 semisynthetic coumermycins described are active antibiotics, and several of them show desirable changes in physical properties from those of the parent coumermycin $\mathrm{A}_{1}$. Comparative activities against Staphylococcus aureus SMITH are reported.

The complete structure of coumermycin $A_{1}$ (I) was first disclosed in a U.S. patent to KAWAGUCHr et al. ${ }^{4}$ It was early recognized that coumermycin $A_{1}$ is a powerful and non-toxic antibiotic with a very good spectrum of activity against gram-positive organisms, as well as considerable activity against a variety of gramnegative bacteria ${ }^{5)}$. In spite of a high degree of in vitro activity, it soon became apparent that because of the insolubility of coumermycin $A_{1}$, even as the sodium salt, parenteral administration was unsatisfactory. Poor oral absorption was also observed. The relationship of coumermycin $A_{1}$ to novobiocin, an antibiotic which is well absorbed orally, was apparent. Novobiocin contains the same association of sugar (noviose, $\mathrm{N}$ ) and coumarin (2-R-amino-4-hydroxy-7-glycosidyl-8-methylcoumarin, C) moieties as is present in coumermycin $A_{1}$ (see Sequence $A$ ), but has a simple carbamoyl function, $-\mathrm{CONH}_{2}$, in place of the 5-methylpyrrolyl-2-carbonyl group (P), and a 4-hydroxy-3-(3-methyl-2-butenyl)benzamide in place of the 3-methylpyrrole-2, 4-dicarboxamide GNP (D-NH-GNP) of coumermycin $\mathrm{A}_{1}$. It seemed that replacement of the 3-methylpyrrole-2,4-dicarboxamide with a simpler amide might result in a structure which would have many of the desirable features of coumermycin $A_{1}$, but which might in addition be more soluble and more readily absorbed via the oral route. The problem therefore reduced to that of removal of the $\underline{D}$ moiety and replacement of it with other acyl functions.

A clue to the displacement of $\underline{D}$ by other acyl functions was provided by the

\footnotetext{
* In the interest of brevity, "acylamido" represents substituted benzoic and the vinylogous cinnamic acid amides.
} 


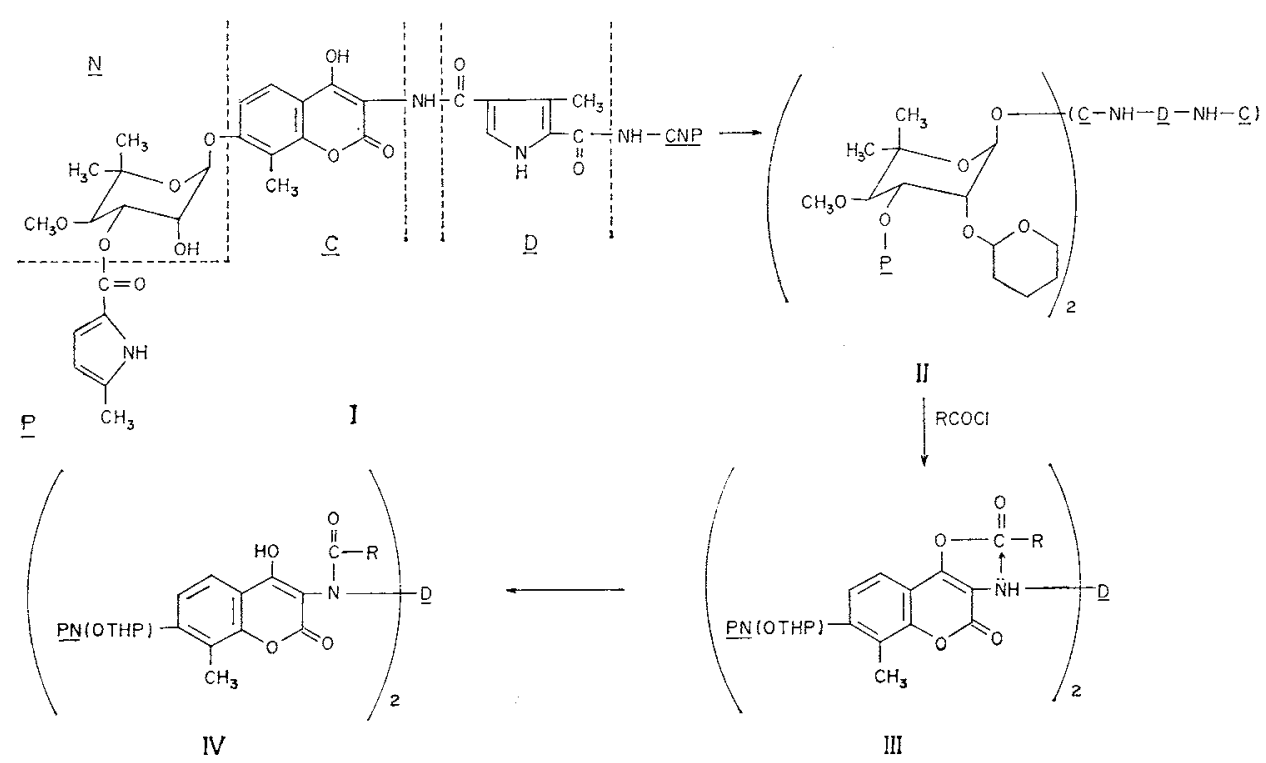

Sequence A, Part 1
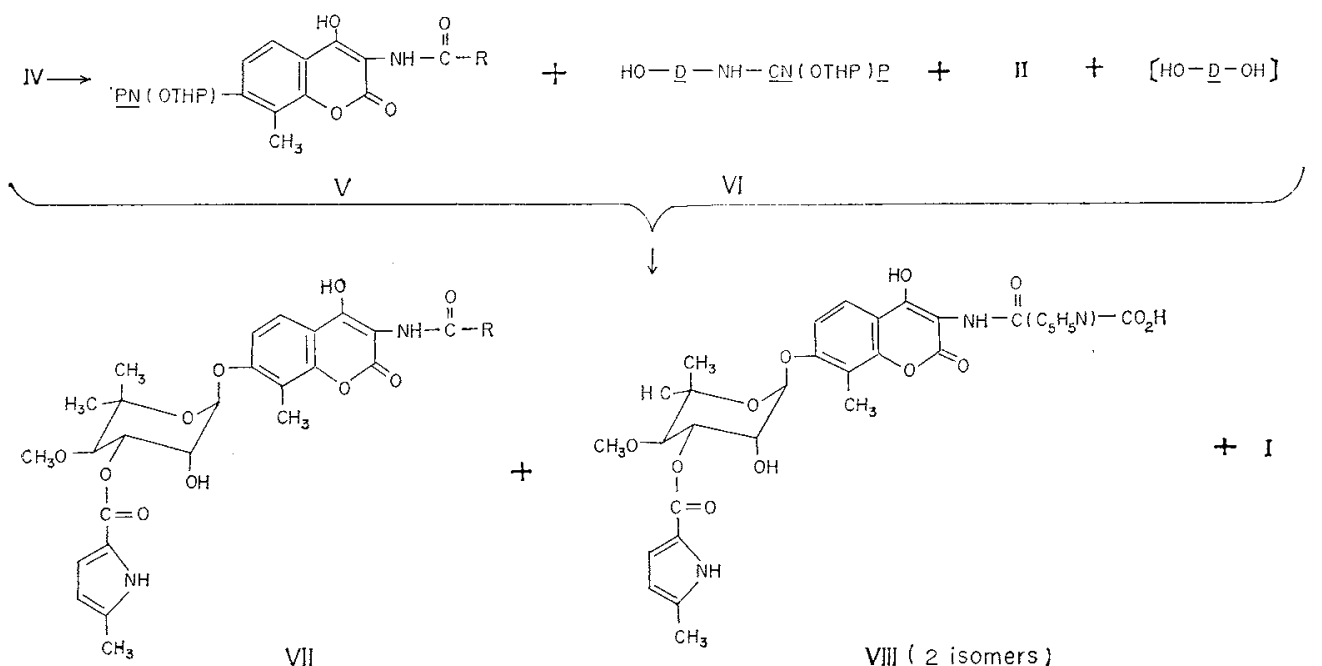

Sequence A. Port 11

work of Hinman et $a l^{3)}$, on the degradation of novobiocin. They noted that novobiocin is cleaved by hot acetic anhydride to give 4-acetoxy-3-(3-methyl-2-butenyl) benzoic acid and an oxazole:

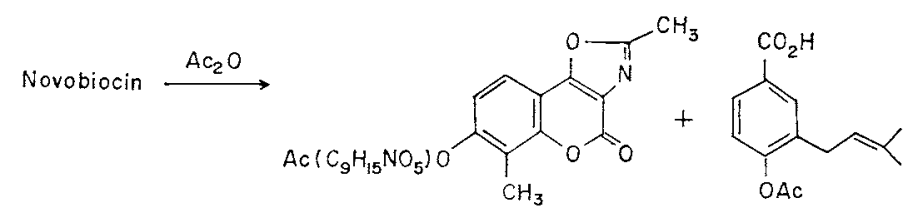

We concluded that it should, in principle, be possible either to find conditions for the acetylation which would result in displacement of the $\mathrm{D}$ moiety, or to hydrolyze 
the oxazole to the 3-acetamido-4-hydroxycoumarin derivative without disruption of the rest of the molecule. In practice, the first-mentioned route was found to be the more practical, but resulted in a derivative in which the 2-hydroxyl of the noviose was blocked with an acetyl group. Derivatives having any sort of block on the noviose-2-hydroxyl have no antibiotic activity. Thus it was necessary to find a blocking group for this hydroxyl which could be readily put on and taken off in high-yield steps. The tetrahydropyranyl group met these requirements quite satisfactorily.

A suspension of coumermycin $A_{1}$ in a mixture of tetrahydrofuran and dihydropyran was treated with a catalytic amount of $p$-toluenesulfonic acid monohydrate and stirred at room temperature for several hours. Work up of the reaction mixture gave an $84 \%$ yield of $2^{\prime}, 2^{\prime}-\mathrm{O}, \mathrm{O}$-ditetrahydropyranylcoumermycin $\mathrm{A}_{1}$ (II), as fine, cubic crystals which decomposed above $200^{\circ} \mathrm{C}$. Coumermycin $\mathrm{A}_{1}$ was shown to be recoverable from the di-THP derivative in high yield by simply stirring it for several hours in methanol solution in the presence of a catalytic amount of $p-$ toluenesulf onic acid monohydrate.

The next proplem was that of finding conditions for the replacement of the $D$ moiety with less complex amides. A review of the literature on $\mathrm{O}$ - and $\mathrm{N}$-acylated 2-aminophenols was very instructive. $B E L L^{1)}$ has reported that alkaline hydrolysis of

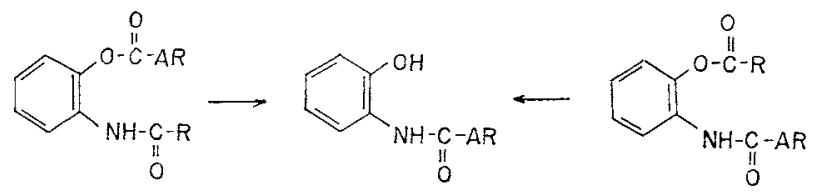

$$
\begin{aligned}
& \text { IXa, R=alkyl } \\
& \mathrm{AR}=\mathrm{C}_{6} \mathrm{H}_{5} \\
& \text { IXb, } \mathrm{R}=\mathrm{C}_{6} \mathrm{H}_{5} \\
& \mathrm{Xa}, \mathrm{AR}=\mathrm{C}_{6} \mathrm{H}_{5} \\
& \mathrm{AR}=\alpha \text {-naphthyl } \\
& \mathrm{XIa}, \mathrm{R}=\text { alkyl } \\
& \mathrm{AR}=\mathrm{C}_{6} \mathrm{H}_{5} \\
& \mathrm{Xb}, \mathrm{AR}=\alpha \text {-naphthy } 1 \\
& \mathrm{XIb}, \mathrm{R}=\mathrm{C}_{6} \mathrm{H}_{5} \\
& \mathrm{AR}=\alpha \text {-naphthy } 1
\end{aligned}
$$

compound IXa and IXb or XIa and XIb leads only to Xa and Xb. Since the product, $X$, is the same regardless of which group is initially on $O$ and which on $N$, BELL postulated the following mechanism (using benzoyl acetyl compounds as examples):

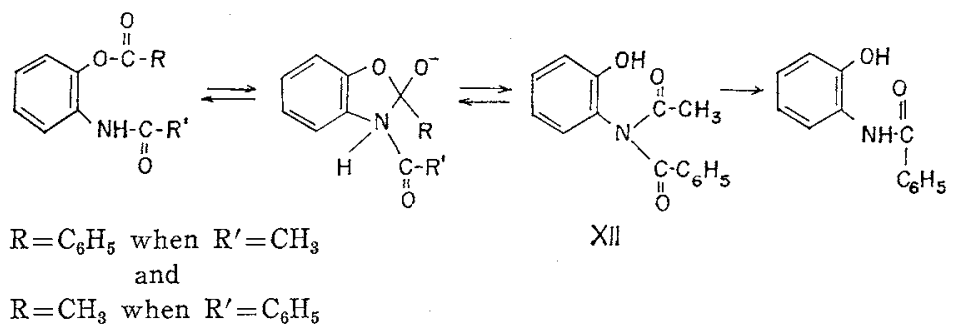

Thus, whichever acyl group of the intermediate XII is the more susceptible to nucleophilic displacement will be the one which is displaced to give the observed ortho-acylaminophenol*. Several conditions were then found which permitted the

* For convenience, this reaction applied to coumermycin will hereinafter be referred to as the transacylation reaction. 
conversion of bis-tetrahydropyranyl coumermycin $A_{1}$ (II) to the imide intermediate IV*. It is believed that this reaction occurs by way of the phenolic ester III, but in all cases III appeared to be very unstable with respect to IV, and IV was the first intermediate beyond II which could be isolated and fully characterized (see Experimetal).

The usual procedure consisted of the addition of four to ten molar equivalents of acid chloride or anhydride to a solution of tetrahydropyranyl coumermycin $\mathrm{A}_{1}$ (II) in pyridine and stirring the mixture at a temperature between $25^{\circ}$ and $115^{\circ} \mathrm{C}$ (reflux) for several hours. An unexpected finding was the beneficial effect of water upon some of the transacylation reactions. In the course of scaling up some of the preparations for pilot plant operation, it was found that very poor yields of transacylation product were obtained when absolutely anhydrous conditions were maintained. Eventually it was found that addition of two moles of water per mole of tetrahydropyranyl coumermycin $A_{1}$ gave the optimum yield of the desired product. (This observation was due to Drs. D. A. Johnson and E.J. Richardson of the Chemical Development Department, Bristol Laboratories.) This observation may be explained as a specific catalysis of the transacylation reaction by hydroxide ion:

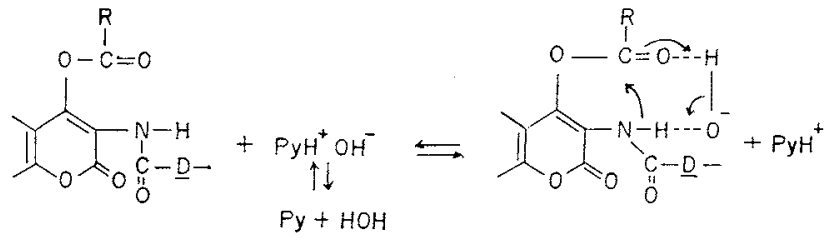

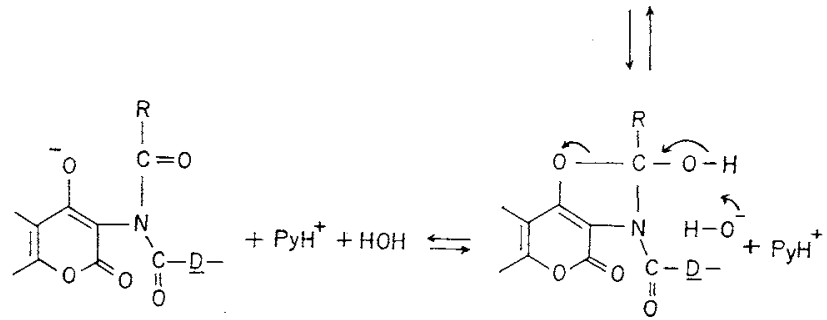

It is clear that pyridine itself could not provide this same kind of specific catalysis. A similar specific catalysis by bicarbonate and dihydrogen phosphate ions in the hydrolysis of 4-hydroxy-butyranalide under very mild conditions has recently been demonstrated ${ }^{2)}$.

In addition to excellent spectroscopic evidence for the proposed structures of the

* Considerable investigation was directed toward finding the best conditions for bis imide (IV) formation and ultimate displacement of the $D$ moiety using the acylating reagents acetic anhydride, benzoic anhydride, and benzoyl chloride in pyridine as a solvent. It was found that the lower temperatures $\left(25^{\circ}\right.$ to $\left.50^{\circ} \mathrm{C}\right)$ and/or smaller molar ratios of reagent to ditetrahydropyranyl coumermycin $A_{1}$ (5 or less) favored the desired reaction. Higher temperatures (at or near reflux) and/or high ratios of reagent to ditetrahydropyranyl coumermycin $A_{1}$ (ca. 10 , or greater) favored formation of 2-methyloxazoles and 2-phenyloxazoles analogous to that produced from novobiocin. As experience was gained with other reagents, it was noted that the most favorable results were obtained under mild conditions when a highly reactive acid chloride, such as $p$-nitrobenzoyl chloride, was used, whereas more drastic conditions gave the best results with reagents of low reactivity. 
bis imides IV (i.e., the absence of amide-II (NH), vibrations near $1540 \mathrm{~cm}^{-1}$ in their infrared spectra, the presence of the methyl group on the $\mathrm{D}$ moiety as shown by its typical $\mathrm{nmr}$ band at $2.4 \mathrm{ppm}$, the expected ratio between aromatic and methyl protons in the integrated $\mathrm{nmr}$ spectra, as well as satisfactory $\mathrm{C}, \mathrm{H}$, and $\mathrm{N}$ analyses), further support for these structures was found in the preparation under very mild conditions, of the PNC-dibenzoylimide. This compound was comparable in all expected respects to the intact bis imides IV. It had no amide-II (NH) band, and the ratio of aromatic to aliphatic protons in its integrated $\mathrm{nmr}$ spectrum was exactly as anticipated for PNC-dibenzoylimide.

As an alternative to the pyridine procedure, tetrahydropyranyl coumermycin $A_{1}$ was dissolved in tetrahydrofuran and treated at $0^{\circ}$ to $25^{\circ} \mathrm{C}$ sequentially with five molar equivalents of triethylamine and four molar equivalents of an acid chloride. In several cases the bis imide IV so produced was readily purified and completely characterized. This procedure was found to be most applicable to acid chlorides which are at least as reactive as benzoyl chloride, while the pyridine procedure was preferred for less reactive acylating agents.

Although many methods were investigated, cleavage of the imide IV was best accomplished by heating in fresh pyridine at about $50^{\circ} \mathrm{C}$ for several hours. Although some imide cleavage could be demonstrated during the acylation step in the pyridine procedure, it appeared to be inhibited in some instances by the presence of pyridine hydrochloride. Therefore, the imide IV was frequently isolated and put back into fresh pyridine. The product of imide cleavage was always a mixture of the tetrahydropyranyl derivatives of the desired transacylation product $(\mathrm{V})$, a coumermycin $A_{1}$ tetrahydropyranyl fragment (VI), from which one of the PNC-NH-groupings had been cleaved, and ditetrahydropyranylcoumermycin $A_{1}$ (II). The mixture could be fractionated at this stage in order to isolate and characterize $V$ and VI (which was done in some cases in order to validate the reaction scheme). In most cases, however, it was found to be more practical to remove the tetrahydropyranyl blocking groups from this mixture of products by exchange with anhydrous methanol at room temperature, catalyzed by $p$-toluenesulfonic acid monohydrate. The second cleavage product then consisted of a mixture of the new antibiotic (VII), PNC-NH-D-OH (VIII), and small amounts of coumermycin $A_{1}(\mathrm{I})$. The components were separated from each, other by fractional precipitation from ethyl acetate with Skellysolve B, by Craig counter-current distribution, or by column chromatography over silica gel or Sephadex LH20.

The preparation of 3-cinnamamido-4-hydroxy-8-methyl-7-[3-O-(5-methylpyrrolylcarbonyl)noviosyloxy]coumarin (compound 59) and analogs substituted on the aromatic ring or on the vinyl group proceeded readily from the corresponding cinnamoyl chlorides via procedures A or B, Table 1. This was as anticipated, since the properties of cinnamic acids parallel closely those of benzoic acids. Therefore no specific examples have been included in the Experimental. It should be noted, however, that the 2- and 4-hydroxy compounds (Nos. 63 and 64) were prepared from 2- and 4acetoxycinnamoyl chlorides. After the transacylation reaction (at $50^{\circ} \mathrm{C}$ for 24 hours) 
the crude product was subjected to the tetrahydropyranyl cleavage reaction directly. The desired products, isolated by fractional precipitation from ethyl acetate with Skellysolve B, were found to be the hydroxy compounds. It is assumed that cleavage of the acetoxy function occurred simultaneously with cleavage of the tetrahydropyranyl groups.

In all cases the purity of the new antibiotic (VII) was assessed by elemental analyses, infrared and $\mathrm{nmr}$ spectra, bioautograph, and/or coumermycin $A_{1}$ content by nickel II complex analysis*. Elemental analyses were only moderately sensitive to impurities such as VIII and I, and many samples were found to be discrete hydrates
Table 1. MIC's of PNC-NH- $\stackrel{\mathrm{C}}{\mathrm{C}}-\mathrm{C}_{6} \mathrm{H}_{5}$ (Compound No. 1) and coumermycin $A_{1}$

\begin{tabular}{l|r|c}
\hline \multicolumn{1}{c|}{ Organism } & $\begin{array}{c}\text { Compound } \\
\text { No. } 1^{*}\end{array}$ & $\begin{array}{c}\text { Coumer- } \\
\text { mycin } A_{1}^{*}\end{array}$ \\
\hline Diplococcus pneumoniae & 1.5 & 0.16 \\
Streptococcus pyogenes & 1.6 & 0.13 \\
Staphylococcus aureus SmITH & 0.25 & 0.0013 \\
Staphylococcus aureus SmITH & 8.8 & 5.2 \\
$\quad$ plus 50\% human serum & & \\
Staphylococcus aureus & 12.5 & 3.1 \\
$\quad$ novobiocin resistant & & \\
Staphylococcus aureus & 2.7 & 0.67 \\
$\quad$ coumermycin $A_{1}$ resistant & $>100$ & 4.4 \\
Proteus morganii & $>100$ & 2.2 \\
Proteus mirabilis & $>100$ & 10.5 \\
E. coli JuHL & $>100$ & 3.7 \\
Salmonella enteritidis & $>100$ & 5.2 \\
Salmonella typhosa & 100 & 1.3 \\
Klebsiella pneumoniae & $>100$ & 12.5 \\
Pseudomonas aeruginosa & \\
\hline
\end{tabular}

* Geometric mean of five determinations. We thank Drs. D. R. Chisholm, M. Misiek, and K. E. Price of the Microbiology Research Department of Bristol Laboratories for the microbiological data in Tables 1,2 and 3.

having from 0.5 to 4 moles of $\mathrm{H}_{2} \mathrm{O}$ per mole of compound. Hydration was supported by elemental analysis data and independent KARL FISCHER moisture determinations. Infrared spectra were generally very much alike from one compound to another. The nmr spectra, on the other hand, were very informative. The resonances for $\mathrm{CH}_{3} \mathrm{O}^{-}$, two types of $\mathrm{CH}_{3}^{-}, \mathrm{H}_{1}$ and $\mathrm{H}_{2}$ of the noviose moiety, and $\mathrm{H}_{\mathrm{AR}}$ of the coumarin and the new amide $\mathrm{R}$ were usually sharp, well separated, and integrated with correct ratios only when the compounds were very pure by other criteria. Bioautographs were run on samples which had been subjected to paper-strip

* Method developed and analyses carried out by P. Monteleone, Chemical Control Department, Bristol Laboratories.

Procedure.

Reagent: 0.001 × $\mathrm{NiSO}_{4} \cdot 5 \mathrm{H}_{2} \mathrm{O}$ in DMSO-Dissolve approximately $262 \mathrm{mg}$ of pulverized $\mathrm{NiSO}_{4}$. $5 \mathrm{H}_{2} \mathrm{O}$ in one liter of spectrograde dimethylsulfoxide by stirring or shaking for approximately 4 hours. Filter through a Whatman $\# 2$ paper to obtain a clear solution.

Standard: Dissolve $10.0 \mathrm{mg}$ of coumermycin $A_{1}$ in $50 \mathrm{ml}$ of DMSO. Pipet $0.50 \mathrm{ml}$ of this solution into a $10 \mathrm{ml}$ volumetric flask, and dilute to volume with $0.001 \mathrm{M} \mathrm{NiSO}$ reagent. Measure the absorbance of this solution at $420 \mathrm{~m} \mu$ in a $1 \mathrm{~cm}$ cell using $0.001 \mathrm{M} \mathrm{NiSO}{ }_{4}$ reagent as a blank.

Sample: Weigh $10.0 \mathrm{mg}$ portions of sample into each of two $10 \mathrm{ml}$ volumetric flasks. Dissolve and dilute one to volume with $0.001 \mathrm{M} \mathrm{NiSO}_{4}$ reagent, and the other with DMSO (Sample Blank. For most of the compounds assayed, as for coumermycin $A_{1}$ itself, the contribution to the absorbance at $420 \mathrm{~m} \mu$ by the uncomplexed material was negligible). Measure the absorbance in the same way as the standard.

Calculation: \% Apparent coumermycin $A_{1}=$

$$
\frac{\left(\mathrm{mg} \text { of coumermycin } A_{1}\right. \text { std.)(Abs. of sample-Abs. of blank) }}{\text { (Abs. of std.)(mg of sample) }}
$$

Sensitivity: Using the test conditions described above, as little as $0.1 \%$ coumermycin $A_{1}$ may be determined. 
0

Table 2. $\mathrm{CD}_{50}$ ' $\mathrm{s}^{\mathrm{a})}$ of $\underline{\mathrm{PNC}}-\mathrm{NH}-\stackrel{\mathrm{C}}{\mathrm{C}}-\mathrm{C}_{6} \mathrm{H}_{5}$ (Compound No. 1) and coumermycin $\mathrm{A}_{1}$

\begin{tabular}{|c|c|c|c|}
\hline Organism & Route & Compound No. 1 & Coumermycin $A_{1}$ \\
\hline \multirow{2}{*}{ Staphylococcus aureus Sмттн } & $\operatorname{im}$ & 3.3 & 0.37 \\
\hline & oral & 3.7 & 10.3 \\
\hline \multirow[t]{2}{*}{ Staphylococcus aureus $1633-2$} & $\mathrm{im}$ & 9.0 & 2.5 \\
\hline & oral & 44 & $>400$ \\
\hline \multirow[t]{2}{*}{ Streptococcus pyogenes A } & $\mathrm{im}$ & 74 & 65 \\
\hline & oral & $110^{b)}$ & $>400^{b)}$ \\
\hline \multirow[t]{2}{*}{ Diplococcus pneumoniae } & $\mathrm{im}$ & $54^{\mathrm{b})}$ & \\
\hline & oral & $107^{b)}$ & $>400^{\mathrm{b})}$ \\
\hline
\end{tabular}

a) Average of three or more determinations.

b) Two treatments with the indicated dose.

Table 3. Semisynthetic coumermycins PNC-NH-C- - R, VII

\begin{tabular}{|c|c|c|c|c|c|c|c|c|}
\hline \multirow{2}{*}{$\begin{array}{c}\text { Com- } \\
\text { pound } \\
\text { No. }\end{array}$} & \multirow{2}{*}{$\mathrm{R}$} & \multirow{2}{*}{ Method ks) } & \multirow{2}{*}{$\begin{array}{c}\text { MIC } \\
\text { Ratio } \text { ? }\end{array}$} & \multirow{2}{*}{$\underset{{ }^{\circ} \mathrm{C}}{\mathrm{dec}} \mathrm{pt.}$} & \multicolumn{2}{|c|}{$\% \quad \mathrm{C}$} & \multicolumn{2}{|c|}{$\% \quad \mathrm{H}$} \\
\hline & & & & & calc. ${ }^{\mathrm{h}\rangle}$ & found & calc. & found \\
\hline 1 & $\left.\mathrm{C}_{6} \mathrm{H}_{5} \mathrm{~d}\right) \quad\left(\mathrm{Na}^{+}\right.$salt $)$ & A & 1.0 & 265 & 60.59 & 60.18 & 5.08 & 5.06 \\
\hline 2 & $2-\mathrm{CH}_{3} \mathrm{C}_{6} \mathrm{H}_{4}$ & A & 1.0 & 234 & 63.36 & 63.44 & 5.65 & 5.86 \\
\hline 3 & $3-\mathrm{CH}_{3} \mathrm{C}_{6} \mathrm{H}_{4}$ & A & 1.0 & 195 & 63.36 & 63.42 & 5.65 & 5.65 \\
\hline 4 & 4- $\mathrm{CH}_{3} \mathrm{C}_{6} \mathrm{H}_{4}{ }^{a}$ ) & A & 1.0 & 239 & 62.42 & 62.59 & 5.73 & 5.77 \\
\hline 5 & $3,5-\left(\mathrm{CH}_{3}\right)_{2} \mathrm{C}_{6} \mathrm{H}_{3}$ & $A$ & 0.50 & 223 & 63.86 & 63.53 & 5.84 & 6.19 \\
\hline 6 & $2,4,6-\left(\mathrm{CH}_{3}\right)_{3} \mathrm{C}_{6} \mathrm{H}_{2}$ & B & 0.50 & 161 & 64.34 & 64.48 & 6.03 & 6.45 \\
\hline 7 & $2-\mathrm{C}_{6} \mathrm{H}_{5} \mathrm{CH}_{2} \mathrm{C}_{6} \mathrm{H}_{4}{ }^{\theta 7}$ & B & 1.0 & 187 & 62.71 & 62.85 & 5.95 & 5.79 \\
\hline 8 & 1-naphthyl & C & 1.0 & 177 & 65.41 & 65.02 & 5.33 & 5.59 \\
\hline 9 & $2-\mathrm{CF}_{3} \mathrm{C}_{6} \mathrm{H}_{4}$ & B & 1.0 & 153 & 58.18 & 58.07 & 4.88 & 5.03 \\
\hline 10 & $\left.3-\mathrm{CF}_{3} \mathrm{C}_{6} \mathrm{H}_{4}{ }^{\mathrm{c}}\right)$ & B & 1.0 & 141 & 55.89 & 55.98 & 4.98 & 4.84 \\
\hline 11 & $4-\mathrm{CF}_{3} \mathrm{C}_{6} \mathrm{H}_{4}$ & B & 1.0 & 157 & 58.18 & 58.15 & 4.88 & 5.19 \\
\hline 12 & $4-\mathrm{H}_{2} \mathrm{NC}_{6} \mathrm{H}_{4}$ & $\mathrm{E}$ & 0.50 & 170 & 61.28 & 61.53 & 5.47 & 5.73 \\
\hline 13 & $3,5-\left(\mathrm{H}_{2} \mathrm{~N}\right)_{2} \mathrm{C}_{6} \mathrm{H}_{3}{ }^{2)}$ & E & 2.0 & 175 & 58.94 & 58.90 & 5.59 & 5.88 \\
\hline 14 & $4-\mathrm{O}_{2} \mathrm{NC}_{6} \mathrm{H}_{4}$ & $A$ & 0.25 & 165 & 58.40 & 58.58 & 4.90 & 5.45 \\
\hline 15 & $3,5-\left(\mathrm{O}_{2} \mathrm{~N}\right)_{2} \mathrm{C}_{6} \mathrm{H}_{3}$ & B & $>4$ & 169 & 54.54 & 54.74 & 4.43 & 4.99 \\
\hline 16 & $4-\mathrm{HOC}_{6} \mathrm{H}_{4}$ & C & 8 & 190 & 61.18 & 60.94 & 5.30 & 5.39 \\
\hline 17 & $2,4-(\mathrm{HO})_{2} \mathrm{C}_{6} \mathrm{H}_{3}$ & B & 4.0 & 184 & 59.61 & 59.36 & 5.16 & 5.62 \\
\hline 18 & $2,6-(\mathrm{HO})_{2} \mathrm{C}_{6} \mathrm{H}_{3}$ & B & 10 & 172 & 59.61 & 59.90 & 5.17 & 5.30 \\
\hline 19 & 4- $\mathrm{CH}_{3} \mathrm{OC}_{6} \mathrm{H}_{5}$ & A & 1.0 & 170 & 61.74 & 61.86 & 5.51 & 5.59 \\
\hline 20 & $2,4-\left(\mathrm{CH}_{3} \mathrm{O}\right)_{2} \mathrm{C}_{6} \mathrm{H}_{3}$ & B & 2.0 & 140 & 60.72 & 61.00 & 5.56 & 5.68 \\
\hline 21 & $2,6-\left(\mathrm{CH}_{3} \mathrm{O}\right)_{2} \mathrm{C}_{6} \mathrm{H}_{3}$ & B & 0.25 & 144 & 59.81 & 59.69 & 5.61 & 5.69 \\
\hline 22 & $3,4,5-\left(\mathrm{CH}_{3} \mathrm{O}\right)_{3} \mathrm{C}_{6} \mathrm{H}_{2}$ & A & 2.0 & 126 & 59.04 & 58.85 & 5.68 & 5.77 \\
\hline 23 & $4-\mathrm{C}_{6} \mathrm{H}_{5} \mathrm{OCH}_{2} \mathrm{CH}_{2} \mathrm{OC}_{6} \mathrm{H}_{4}$ & B & 0.064 & 144 & 64.27 & 64.35 & 5.53 & 5.67 \\
\hline 24 & $2-\mathrm{FC}_{6} \mathrm{H}_{4}$ & B & 4 & 148 & 60.98 & 61.28 & 5.12 & 5.46 \\
\hline 25 & $4-\mathrm{FC}_{6} \mathrm{H}_{4}$ a) & A & 4 & 110 & 60.09 & 60.38 & 5.21 & 5.15 \\
\hline 26 & $3-\mathrm{ClC}_{6} \mathrm{H}_{4}$ & $\mathrm{~B}$ & 2.0 & 140 & 59.38 & 59.36 & 4.98 & 5.53 \\
\hline 27 & $4-\mathrm{ClC}_{6} \mathrm{H}_{4}$ & B & 2.0 & 250 & 59.38 & 59.32 & 4.98 & 5.33 \\
\hline 28 & $2,5-\mathrm{Cl}_{2} \mathrm{C}_{6} \mathrm{H}_{3}$ d) & B & 1.0 & 144 & 55.66 & 55.74 & 4.67 & 5.00 \\
\hline 29 & $2,6-\mathrm{Cl}_{2} \mathrm{C}_{6} \mathrm{H}_{3}$ & B & 0.50 & 161 & 56.28 & 56.38 & 4.57 & 5.22 \\
\hline 30 & $3,4-\mathrm{Cl}_{2} \mathrm{C}_{6} \mathrm{H}_{3}$ b) & B & 0.25 & 159 & 54.79 & 54.49 & 4.75 & 4.84 \\
\hline 31 & $3,5-\mathrm{Cl}_{2} \mathrm{C}_{6} \mathrm{H}_{3}$ & B & 2.0 & 150 & 56.28 & 56.81 & 4.57 & 4.60 \\
\hline
\end{tabular}


Table 3 (continued)

\begin{tabular}{|c|c|c|c|c|c|c|c|c|}
\hline \multirow{2}{*}{$\begin{array}{c}\text { Com- } \\
\text { pound } \\
\text { No. }\end{array}$} & \multirow{2}{*}{$\mathrm{R}$} & \multirow{2}{*}{ Method k) } & \multirow{2}{*}{$\begin{array}{c}\text { MIC } \\
\text { Ratio s) }\end{array}$} & \multirow{2}{*}{${ }_{{ }^{\circ} \mathrm{C}}^{\operatorname{dec} .} \mathrm{pt}$} & \multicolumn{2}{|c|}{$\% \mathrm{C}$} & \multicolumn{2}{|c|}{$\% \quad \mathrm{H}$} \\
\hline & & & & & calc. ${ }^{\text {h) }}$ & found & calc. & found \\
\hline 32 & $4-\mathrm{BrC}_{6} \mathrm{H}_{4}$ & A & 1.0 & 165 & 55.44 & 55.02 & 4.66 & 4.65 \\
\hline 33 & $4-\mathrm{IC}_{6} \mathrm{H}_{4}$ 2) & A & 1.0 & 125 & 51.38 & 51.36 & 4.43 & 4.70 \\
\hline 34 & $2-\mathrm{HO}-3-\mathrm{CH}_{3} \mathrm{C}_{6} \mathrm{H}_{3}$ a) & $\mathrm{B}$ & 1.0 & 173 & 60.84 & 61.10 & 5.59 & 5.84 \\
\hline 35 & $3-\mathrm{HO}-4-\mathrm{CH}_{3} \mathrm{C}_{6} \mathrm{H}_{3}$ & B & 1.0 & 180 & 61.73 & 61.37 & 5.50 & 5.63 \\
\hline 36 & $4-\mathrm{CH}_{3}-3-\mathrm{NO}_{2} \mathrm{C}_{6} \mathrm{H}_{3}$ a) & $\mathrm{C}$ & 0.50 & 246 & 58.17 & 58.21 & 5.18 & 5.16 \\
\hline 37 & $3-\mathrm{NH}_{2}-4-\mathrm{CH}_{3} \mathrm{C}_{6} \mathrm{H}_{3}$ & $\mathrm{E}$ & 1.0 & 225 & i & & & \\
\hline 38 & $4-\mathrm{NH}_{2}-3 \mathrm{EtC}_{6} \mathrm{H}_{3}$ & $\mathrm{E}$ & 0.0016 & 202 & 62.35 & 62.12 & 5.87 & 6.29 \\
\hline 39 & $\left.2-\mathrm{HO}-3 \mathrm{NO}_{2} \mathrm{C}_{6} \mathrm{H}_{3} \mathrm{f}\right)$ & $\mathrm{D}$ & 8.0 & $>350$ & 50.47 & 50.48 & 5.31 & 5.06 \\
\hline 40 & $4-\mathrm{HO}-3-\mathrm{NO}_{2} \mathrm{C}_{6} \mathrm{H}_{3}$ & B & 40 & 162 & 56.97 & 57.45 & 4.78 & 5.33 \\
\hline 41 & $3-\mathrm{HO}-4-\mathrm{NO}_{2} \mathrm{C}_{6} \mathrm{H}_{3}$ b) & $\mathrm{C}$ & 4.0 & 177 & 55.43 & 55.71 & 4.95 & 4.90 \\
\hline 42 & $3-\mathrm{NH}_{2}-4-\mathrm{HOC}_{6} \mathrm{H}_{3}$ a) & $\mathrm{E}$ & 4.0 & 222 & 58.85 & 59.07 & 5.41 & 5.94 \\
\hline 43 & $4-\mathrm{NH}_{2}-3-\mathrm{HOC}_{6} \mathrm{H}_{3}$ b) & $\mathrm{E}$ & 2.0 & 232 & 58.03 & 58.18 & 5.50 & 5.86 \\
\hline 44 & $\left.4-\mathrm{HO}-3-\left(\mathrm{Et}_{2} \mathrm{CHCONH}\right) \mathrm{C}_{6} \mathrm{H}_{3} \mathrm{~b}\right)$ & $\mathrm{F}$ & 8.0 & 146 & 60.07 & 60.39 & 6.13 & 6.09 \\
\hline 45 & 4- $\left.\mathrm{HO}-3-\left(\mathrm{C}_{6} \mathrm{H}_{5} \mathrm{CONH}\right) \mathrm{C}_{6} \mathrm{H}_{3}{ }^{e}\right)$ & $\mathrm{F}$ & 2.0 & 147 & 59.06 & 59.17 & 5.48 & 5.55 \\
\hline 46 & $3-\mathrm{CH}_{3} \mathrm{O}-2-\mathrm{NO}_{2} \mathrm{C}_{6} \mathrm{H}_{3}{ }^{a}$ & $\mathrm{D}$ & 8.0 & 184 & 56.80 & 56.90 & 5.06 & 5.07 \\
\hline 47 & $\left.3-\left(n \mathrm{C}_{4} \mathrm{H}_{9} \mathrm{O}\right)-4-\mathrm{NO}_{2} \mathrm{C}_{6} \mathrm{H}_{3} \mathrm{~d}\right)$ & $A$ & 0.12 & 198 & 56.36 & 56.16 & 5.81 & 5.78 \\
\hline 48 & $2-\mathrm{HO}-4-\mathrm{EtOC}_{6} \mathrm{H}_{3}$ & $B$ & 1.0 & 182 & 60.73 & 60.94 & 5.56 & 5.78 \\
\hline 49 & 2-EtO-1-naphthyl & B & 0.25 & 175 & 64.81 & 64.89 & 5.44 & 5.86 \\
\hline 50 & $\left.4-\mathrm{HO}-3,5-\mathrm{I}_{2} \mathrm{C}_{6} \mathrm{H}_{2}{ }^{\mathrm{j}}\right)$ & $A$ & 4.0 & 204 & 43.27 & 45.08 & 3.51 & 4.11 \\
\hline 51 & $2,6-\mathrm{Cl}_{2} \mathrm{C}_{6} \mathrm{H}_{3}-{ }_{\mathrm{O}} \|_{\mathrm{CH}_{3}}$ & $\mathrm{~B}$ & 2.0 & 153 & 56.61 & 56.64 & 4. 48 & 4.90 \\
\hline 52 & $\mathrm{C}_{6} \mathrm{H}_{5}{ }_{\mathrm{O}}^{\mathrm{N}} \mathrm{CH}_{3}$ & $B$ & 4.0 & 122 & 61.58 & 61.32 & 5.32 & 5.47 \\
\hline 53 & & A & 0.25 & 243 & 54.06 & 54.24 & 4.87 & 4.71 \\
\hline 54 & & A & 2.0 & 258 & 56.08 & 56.09 & 4.87 & 5.20 \\
\hline 55 & 2-Pyridyl b) & $\mathrm{G}$ & 2.0 & 140 & 58.91 & 59.10 & 5.43 & 5.30 \\
\hline 56 & $\left.\stackrel{\mathrm{H}_{3} \mathrm{C}}{\mathrm{N}}\right|_{\mathrm{O}}=\mathrm{CH}_{3}$ & B & 0.50 & 231 & 58.91 & 58.94 & 5.43 & 5.63 \\
\hline 57 & 2-Quinolyl & $\mathrm{C}$ & 2.0 & 155 & 63.44 & 63.82 & 5.17 & 5.34 \\
\hline 58 & & $\mathrm{C}$ & 4.0 & 153 & 64.80 & 64.50 & 5.73 & 5.91 \\
\hline 59 & $\mathrm{C}_{6} \mathrm{H}_{5} \mathrm{CH}=\mathrm{CH}-$ & A & 1.0 & 201 & 64.07 & 63.69 & 5.54 & 5.31 \\
\hline 60 & $4-\left(\mathrm{CH}_{3}\right)_{2} \mathrm{CHC}_{6} \mathrm{H}_{4} \mathrm{CH}=\mathrm{CH}-$ & $\mathrm{B}$ & 2.0 & 163 & 65.44 & 65.51 & 6.10 & 6.48 \\
\hline 61 & $2-\mathrm{CH}_{3} \mathrm{OC}_{6} \mathrm{H}_{4} \mathrm{CH}=\mathrm{CH}-$ & A & 1.0 & 180 & 62.95 & 63.30 & 5.59 & 5.22 \\
\hline 62 & $\left.4-\mathrm{CH}_{3} \mathrm{OC}_{6} \mathrm{H}_{4} \mathrm{CH}=\mathrm{CH}-{ }^{2}\right)$ & A & 0.12 & 195 & 62.09 & 61.95 & 5.67 & 5.64 \\
\hline 63 & $2-\mathrm{HOC}_{6} \mathrm{H}_{4} \mathrm{CH}=\mathrm{CH}-{ }^{a)}$ & A & 0.50 & 190 & 61.57 & 61.77 & 5.48 & 4.93 \\
\hline 64 & $4-\mathrm{HOC}_{6} \mathrm{H}_{4} \mathrm{CH}=\mathrm{CH}-{ }^{\text {a) }}$ & $\mathrm{A}$ & 0.25 & 196 & 61.57 & 61.95 & 5.48 & 5.75 \\
\hline
\end{tabular}


Table 3 (continued)

\begin{tabular}{|c|c|c|c|c|c|c|c|c|}
\hline \multirow{2}{*}{$\begin{array}{l}\text { Com- } \\
\text { pound } \\
\text { No. }\end{array}$} & \multirow{2}{*}{$\mathrm{R}$} & \multirow{2}{*}{ Method ks } & \multirow{2}{*}{$\begin{array}{c}\text { MIC } \\
\text { Ratio g) }\end{array}$} & \multirow{2}{*}{${ }_{{ }^{\circ} \mathrm{C}}^{\text {dec. pt. }}$} & \multicolumn{2}{|c|}{$\% \mathrm{C}$} & \multicolumn{2}{|c|}{$\% \quad \mathrm{H}$} \\
\hline & & & & & calc. ${ }^{b)}$ & found & calc. & found \\
\hline 65 & $3-\mathrm{O}_{2} \mathrm{NC}_{6} \mathrm{H}_{4} \mathrm{CH}=\mathrm{C}(\mathrm{Et})-$ & $B$ & 2.0 & 150 & 60.77 & 61.06 & 5.39 & 5.55 \\
\hline 66 & $4-\mathrm{CH}_{3} \mathrm{OC}_{6} \mathrm{H}_{4} \mathrm{CH}=\mathrm{C}\left(4-\mathrm{CH}_{3} \mathrm{OC}_{6} \mathrm{H}_{4}\right)-$ & $B$ & $>4$ & 182 & 65.24 & 65.31 & 5.61 & 5.78 \\
\hline 67 & $\mathrm{C}_{6} \mathrm{H}_{5} \mathrm{CH}=\mathrm{CCl}$ & $\mathrm{B}$ & 1.0 & 196 & 60.69 & 60.75 & 5.09 & 5.39 \\
\hline
\end{tabular}
a) hemihydrate.
b) monohydrate.
c) sesquihydrate.
d) dihydrate.
e) $2.5 \mathrm{H}_{2} \mathrm{O}$.
f) tetrahydrate.
g) Minimum inhibitory concentration of compound vs. Staphylococcus aureus Smir in Antibiotic Assay Broth (BBL), pH 6.0, divided by the MIC for Compound 1 in the same medium. Compound 1 had an MIC of from $0.0125 \mathrm{mcg} / \mathrm{ml}$ to $0.05 \mathrm{mcg} / \mathrm{ml}$ under these conditions.
h) In nearly all cases, nitrogen analyses which were within $\pm 0.3 \%$ of the theoretical values were also obtained.
i) Sample was too small for analysis but was made by reduction of compound 36 , which was very pure. j) Elemental analyses always indicated that $c a .10 \%$ of the iodine present in starting acid was
lost during reaction.
k) Methods (details in Experimental): A, acid chloride in pyridine at $25^{\circ}$ to $50^{\circ} \mathrm{C}$, several hours; $\mathrm{B}$, acid chloride in pyridine at reflux, $1 \sim 3$ hours; $\mathrm{C}$, acid chloride in THF-triethylamine at $0 \sim 65^{\circ} \mathrm{C}$; D, mixed anhydride in THF-triethylamine at $0 \sim 65^{\circ} \mathrm{C}$; E, catalytic reduction of one or more nitro groups; $\mathrm{F}$, acylation of compound $42 ; \mathrm{G}$, acid chloride in THF-pyridine at $25^{\circ} \mathrm{C}$, several hours.

chromatography*, which gave good separations of the new antibiotics VII from coumermycin $A_{1}$. Calibration of the paper chromatography-bioautograph system with coumermycin $A_{1}$ permitted ready quantitation of $0.5 \%$ or more contamination of the sample by coumermycin $A_{1}$ in most cases. Samples were judged to be pure on a bioactivity basis when the coumermycin $A_{1}$ content was found to be $\leqq 0.5 \%$ (weight).

All of the compounds VII were found to be active antibiotics. Their structureactivity relationships will be described in detail in a forthcoming publication. The in vitro activities of compound No. 1 and coumermycin $A_{1}$ against a variety of organisms are compared in Table 1. Table 2 is particularly informative, in that it shows that in spite of coumermycin $A_{1}$ 's consistently superior activity in vitro, compound No. 1 has considerably greater therapeutic activity via the oral route. This is particularly significant because coumermycin $\mathrm{A}_{1}$ cannot be employed via the intramuscular route because of its irritation liability and poor absorption from the site of injection in higher animals. The physical properties and relative in vitro activities of the semisynthetic coumermycins are reported in Table 3.

\footnotetext{
* Procedure due to C. A. ClaAridge and V. Z. Rossomano, Microbiology Research Department, Bristol Laboratories. Chromatography was carried out on $1 / 2$ " strips of $S \&$ S589 Blue Ribbon paper in the solvent system acetone: $0.1 \mathrm{M}$ triethanolamine adjusted to $\mathrm{pH} 7.0$ with glacial acetic acid: $2: 3$. The new antibiotics had Rf's of $0.8 \sim 0.9$, while coumermycin $A_{1}$ had $R f 0.3 \sim$ 0.4. Bioautography was done on agar plates seeded with Staphylococcus aureus ATCC 6538 P.
} 


\section{Experimental ${ }^{*, \dagger}$}

$2^{\prime}, 2^{\prime}-\mathrm{O}, \mathrm{O}-$ Ditetrahydropyranylcoumermycin $\mathrm{A}_{1}$ (II).

Finely ground coumermycin $A_{1}, 5.5 \mathrm{~g}$ was stirred with $50 \mathrm{ml}$ of dihydropyran and a trace of $p$-toluenesulfonic acid monohydrate $(2 \sim 3 \mathrm{mg})$ was added. The mixture was stirred in a stoppered flask under anhydrous conditions at $25^{\circ} \mathrm{C}$ for $3 \frac{1}{2}$ hours, dissolution of the solid being complete after 2 hours.

The solution was evaporated to dryness in vacuo at minimum temperature (below $\left.40^{\circ} \mathrm{C}\right)$ and the residue remaining was dissolved in boiling acetone $(30 \mathrm{ml})$. Hot ethanol was slowly added with stirring and heating until $100 \mathrm{ml}$ had been added. The product crystallized upon cooling overnight to yield $5.3 \mathrm{~g}(84 \%)$ of fine cube-like crystals. An additional $810 \mathrm{mg}(13 \%)$ separated as a second crop from the mother liquor after standing at $-5^{\circ} \mathrm{C}$ for 24 hours.

A sample $(1.7 \mathrm{~g})$ was recrystallized twice from acetone-ethanol to give a pure sample $(1.5 \mathrm{~g}), \mathrm{mp}$ (decomposition) above $200^{\circ} \mathrm{C}$.

Analysis. Calculated for $\mathrm{C}_{65} \mathrm{H}_{75} \mathrm{O}_{22} \mathrm{~N}_{5}:$ C $61.06, \mathrm{H} 5.91, \mathrm{~N} 5.47$.

Found: C $61.00, \mathrm{H} 5.83, \mathrm{~N} 5.56$.

Neutral equivalent: Found 623, calculated 634.

Amberlyst $15\left(\mathrm{H}^{+}\right)$resin may be substituted for toluenesulfonic acid as the catalyst in the reaction. In this case, filtration of the catalyst and concentration of the filtrate under anhydrous conditions produces a syrup from which a mixture of mono-, di-, tri-, and tetra-tetrahydropyranyl coumermycin $A_{1}$ may be precipitated as an amorphous solid by triturating in the cold with a minimal amount of dry methanol. Evidence that this was indeed a mixture of the named compounds was initially obtained from thin-layer chromatography on silica gel plates, in a solvent system consisting of $9: 21: 8$ (parts by volume) of methyl acetate : 2 -propanol : concentrated ammonium hydroxide. In this system the $R f$ values are: Coumermycin $A_{1}, 0.3$; monotetrahydropyranyl derivative, $0.45 \sim 0.50$; di-, tri-, and tetra-tetrahydropyranyl derivatives, an elongated zone at $\operatorname{Rf} 0.60$ to 0.70 . Individual tlc of the separated products confirmed these Rf values. A CRAIG countercurrent distribution separation was run on a $15-\mathrm{g}$ sample of the mixture using $\frac{1}{2}$ volume upper phase to 1 volume lower phase from a system of $5: 1: 5: 1$ of $\mathrm{CCl}_{4}: \mathrm{CHCl}_{3}: \mathrm{CH}_{3} \mathrm{OH}$ : $\mathrm{H}_{2} \mathrm{O}$ over 1001 transfers, $97.5 \%$ of the solid being recovered in total. The recoveries from the major concentrations, as determined by ultraviolet absorption at $345 \mathrm{~m} \mu$, were as follows:

$2^{\prime}, 2^{\prime}, 4,4-\mathrm{O}, \mathrm{O}, \mathrm{O}, \mathrm{O}-\mathrm{Tetratetrahydropyranylcoumermycin} \mathrm{A}_{1}$.

The tetra-substituted tetrahydropyranyl ether of coumermycin $A_{1}$ was recovered from tubes 21 through 40 as a pure crystalline solid, $3.68 \mathrm{~g}, \mathrm{mp}$ (decomposition) above $200^{\circ} \mathrm{C}$.

Analysis. Calculated for $\mathrm{C}_{75} \mathrm{H}_{91} \mathrm{~N}_{5} \mathrm{O}_{24}:$ C $62.27, \mathrm{H} 6.34, \mathrm{~N} 4.84$. Found: C $62.03, \mathrm{H} 6.31, \mathrm{~N} 4.94$.

$2^{\prime}, 2^{\prime}, 4-\mathrm{O}, \mathrm{O}, \mathrm{O}-$ Tritetrahydropyranylcoumermycin $\mathrm{A}_{1}$.

The tri-substituted tetrahydropyranyl ether of coumermycin $A_{1}$ was recovered from tubes $41-70$ as a pure crystalline solid, $3.8 \mathrm{~g}, \mathrm{mp}$ (decomposition) above $200^{\circ} \mathrm{C}$.

Analysis. Calculated for $\mathrm{C}_{70} \mathrm{H}_{83} \mathrm{~N}_{5} \mathrm{O}_{23}: \quad \mathrm{C} 61.71, \mathrm{H} 6.14, \mathrm{~N} 5.14$.

Found: C 61.65, H 6.19, N 5.34.

$2^{\prime}, 2^{\prime}-\mathrm{O}, \mathrm{O}-\mathrm{Ditetrahydropyranylcoumermycin} \mathrm{A}_{1}$.

The disubstituted tetrahydropyranyl ether of coumermycin $A_{1}$ was recovered from tubes $71 \sim 100$ as a pure crystalline solid, $1.8 \mathrm{~g}, \mathrm{mp}$ (decomposition) above $200^{\circ} \mathrm{C}$. The

* The authors are indebted to R. L. DeVault, E. R. May, C. D. McDonnell, and C. L. Swanson for many of the preparations reported in this paper; to R. M. Downing and C. M. KALinowskI for elemental analyses; and to D.F. Whitehead and A. L. Vulcano for the infrared and nmr spectra.

$\uparrow$ Melting points are not corrected. 
product was identical in its physical characteristics to previously characterized material.

$2^{\prime}$-O-Monotetrahydropy ranylcoumermycin $\mathrm{A}_{1}$.

The monosubstituted tetrahydropyranyl ether of coumermycin $A_{1}$ was recovered from tubes $101 \sim 130$ as a pure crystalline solid, $1.6 \mathrm{~g}, \mathrm{mp}$ (decomposition) above $200^{\circ} \mathrm{C}$.

$$
\begin{array}{r}
\text { Analysis. Calculated for } \mathrm{C}_{60} \mathrm{H}_{65} \mathrm{~N}_{5} \mathrm{O}_{21} \text { : } \\
\text { Found: } 60.35 \text {, H } 5.66, \mathrm{C} 5.86 .42 \text {, H 5.81, N } 5.83 .
\end{array}
$$

Coumermycin $\mathrm{A}_{1}$ was recovered unreacted from tubes $300 \sim 499,1.5 \mathrm{~g}, \mathrm{mp}$ (decomposition) $240 \sim 245^{\circ} \mathrm{C}$.

3-Benzamido-4-hydroxy-8-methyl-7-[3-O-(5-methyl-2-pyrrolylcarbonyl)noviosyloxy] coumarin. (VII, $\mathrm{R}=\mathrm{C}_{6} \mathrm{H}_{5}$, Compound 1). Method $\mathrm{A}$.

Ditetrahydropyranyl coumermycin $\mathrm{A}_{1}$ (I), $1278 \mathrm{~g}$ (1.00 mole), was dissolved in 16.0 liters of anhydrous pyridine. Water, $36.0 \mathrm{~g}$ (2.00 moles), was added, followed by $480 \mathrm{ml}$ (4.00 moles) of benzoyl chloride. The reaction mixture was protected from atmospheric moisture and stirred at $50^{\circ} \mathrm{C}$ for 22 hours. The solution was concentrated in vacuo to 4.5 liters and was then poured into 50.0 liters of vigorously agitated ice water. The acidity of the mixture was quickly adjusted to 2.0 with $6 \mathrm{~N} \mathrm{HCl}$. The precipitated solid (V, $\mathrm{R}=\mathrm{C}_{6} \mathrm{H}_{5}$, plus VI, plus II, plus D) was removed by filtration, washed thoroughly with cold water, and dried in an oven at $60^{\circ} \mathrm{C}$. The dried intermediate was suspended in 16.0 liters of dry methanol, to which $326 \mathrm{~g}$ ( 1.7 mole) of $p$-toluenesulfonic acid monohydrate was added. The mixture was stirred for 15 hours at $25^{\circ} \mathrm{C}$, then poured into 50.0 liters of vigorously stirred ice water. The crude product was filtered off, washed with water at $\mathrm{pH} 5$ to 7 , and dried in an oven at $60^{\circ} \mathrm{C}$. The title compound was purified by fractional precipitation from ethyl acetate by addition of Skellysolve B. The impurities (I and VIII) precipitated first, after which the title compound was obtained as a cream-colored, crystalline solid, $\mathrm{mp} 230 \sim 235^{\circ} \mathrm{C}$ (d) after softening at $c a \cdot 190^{\circ} \mathrm{C}$. The yield was $425 \mathrm{~g}$.

PNC-NH-D-OH. (VIII, 2 isomers).

Eleven grams of the crude tetrahydropyranyl intermediate V (plus VI, plus II, plus D) was thoroughly mixed with an equal amount of silicic acid (Baker Analyzed, Reagent) and transferred as a slurry in $10 \%$ ethyl acetate $-90 \% n$-hexane to the top of a chromatographic column packed with $600 \mathrm{~g}$ of silicic acid and wet with the same solvent. The column was eluted successively with 3.00 liters of $10 \%$ ethyl acetate, 46.66 liters of $20 \%$ ethyl acetate, 2.82 liters of $30 \%$ ethyl acetate, 6.35 liters of $40 \%$ ethyl acetate, and 3.56 liters of $60 \%$ ethyl acetate. The latter three eluates were combined and concentrated to dryness, yielding $1.98 \mathrm{~g}$ of solid which was stirred with $200 \mathrm{ml}$ of chloroform. The material which failed to dissolve was filtered off and recrystallized from absolute ethanol, $115 \mathrm{mg}$. Integration of the $\mathrm{nmr}$ spectrum revealed the correct ratios of the various types of protons for structure VI.

\section{Analysis. Calculated for $\mathrm{C}_{36} \mathrm{H}_{41} \mathrm{~N}_{3} \mathrm{O}_{13}$ : C 59.75, H 5.71, N 5.81; mol wt, 723.69. Found: C 59.18 , H 5.77, N 5.75; \\ mol wt, 742 (osmometric in tetrahydrofuran).}

This tetrahydropyranyl intermediate, $100 \mathrm{mg}$, was dissolved in a mixture of $8.0 \mathrm{ml}$ of methanol and $2.0 \mathrm{ml}$ of tetrahydrofuran, with gentle warming as required to effect solution. A small amount (ca. $0.3 \mathrm{~g}$ ) of Amberlyst-15 resin $\left(\mathrm{H}^{+}\right)$was added and the mixture was stirred at $25^{\circ} \mathrm{C}$ for 24 hours. The resin was filtered off, washed with a little methanol, and the filtrate was concentrated to dryness in vacuo, leaving a powdery, yellow solid. It was dissolved in $50 \mathrm{ml}$ of hot ethyl acetate and diluted with $115 \mathrm{ml}$ of $n$-hexane. After 22 hours at $4^{\circ} \mathrm{C}$ the tan precipitate was filtered off and dried for analysis, $57 \mathrm{mg}$, $\mathrm{mp} 300^{\circ} \mathrm{C}(\mathrm{d})$.

$$
\text { Analysis. Calculated for } \mathrm{C}_{31} \mathrm{H}_{33} \mathrm{~N}_{3} \mathrm{O}_{12} \text { : C } 58.21, \mathrm{H} 5.21, \mathrm{~N} 6.57 \text {. }
$$

Found: C $58.52, \mathrm{H} 5.84, \mathrm{~N} 6.34$. 
3-(4-Chlorobenzamido)-4-hydroxy-8-methyl-7-[3-O-(5-methyl-2-pyrrolylcarbonyl) noviosyloxy] coumarin. (Compound 27). Method B.

$p$-Chlorobenzoyl chloride, $2.74 \mathrm{~g}$ (15.6 millimoles) was carefully added at $25^{\circ} \mathrm{C}$ to a solution of $5.00 \mathrm{~g}$ (3.91 millimoles) of ditetrahydropyranyl coumermycin $A_{1}$ in $100 \mathrm{ml}$ of freshly-distilled pyridine. The clear solution was refluxed $\left(115^{\circ} \mathrm{C}\right)$ for 2.0 hours and poured into $1250 \mathrm{ml}$ of ice-cold $1.2 \mathrm{~N} \mathrm{HCl}$. After standing at $4^{\circ} \mathrm{C}$ for 15 hours, the tan, amorphous solids were filtered off and washed with four $200 \mathrm{ml}$ portions of water. It was dried to give $5.92 \mathrm{~g}$ of crude product. Removal of the tetrahydropyranyl moieties was effected by dissolving $5.0 \mathrm{~g}$ of the product in $50 \mathrm{ml}$ of $\mathrm{THF}$ and $170 \mathrm{ml}$ of methanol, adding $750 \mathrm{mg}$ of $p$-toluenesulfonic acid monohydrate, and stirring at $25^{\circ} \mathrm{C}$ for 66 hours. The solution was filtered to remove $512 \mathrm{mg}$ of dark, amorphous solid (discarded), the filtrate was diluted with $200 \mathrm{ml}$ of water and concentrated at $40^{\circ} \mathrm{C}$ in vacuo to remove most of the methanol and THF. The pale, golden solid which precipitated was filtered off and dried, $3.25 \mathrm{~g}$. Of this material, $3.0 \mathrm{~g}$ was dissolved in $200 \mathrm{ml}$ of chloroform (warmed gently to dissolve). The solution was cooled to ca. $25^{\circ} \mathrm{C}$ and $486 \mathrm{mg}$ of dark, amorphous solid was filtered off. The filtrate was extracted with five $200 \mathrm{ml}$ portions of $5 \%$ aqueous sodium bicarbonate, which removed (as shown by tlc) p-chlorobenzoic acid, coumermycin $\mathrm{A}_{1}$, and $\mathrm{PNC}-\mathrm{NH}-\mathrm{D}-\mathrm{OH}$. The chloroform solution was dried over sodium sulfate, filtered, and diluted with two increments of Skellysolve $B$ to produce successively an impure fraction ( $344 \mathrm{mg}$ ) and the desired title compound, $310 \mathrm{mg}$.

3-(4-Methyl-3-nitrobenzamido)-4-hydroxy-8-methyl-7-[3-O-(5-methyl-2-pyrrolylcarbonyl)noviosyloxy] coumarin. (Compound 36). Method C.

Ditetrahydropyranyl coumermycin $\mathrm{A}_{1}$ (II), $10.0 \mathrm{~g}$ (7.82 millimoles) was dissolved in $100 \mathrm{ml}$ of tetrahydrofuran (THF) at $25^{\circ} \mathrm{C}$, and $5.50 \mathrm{ml}(3.96 \mathrm{~g}, 39.1$ millimoles $)$ of triethylamine and $0.28 \mathrm{ml}$ (15.6 millimoles) of water were added. To this mixture was added $4.60 \mathrm{ml}$ ( $6.24 \mathrm{~g}, 31.3$ millimoles) of 4-methyl-3-nitrobenzoyl chloride (Frinton Laboratories) and the solution was heated at reflux $\left(65^{\circ} \mathrm{C}\right)$ for 3.5 hours. The solution turned orange during this time. The reaction mixture was cooled at $4^{\circ} \mathrm{C}$ for 15 hours and filtered to remove triethylamine hydrochloride. The filtrate was poured into 1.0 liter of ice water and the solid which separated was filtered off, washed, and thoroughly dried, $14.20 \mathrm{~g}$ of peach-colored solid. The infrared and $\mathrm{nmr}$ spectra showed that this intermediate was the intact bis imide IV ( $\mathrm{R}=4$-methyl-3-nitrophenyl). Cleavages of the imide in warm pyridine (see procedure $\mathrm{D}$ ) and of the tetrahydropyranyl groups in methanol-acetone with $p$-toluenesulfonic acid catalysis were carried out in the usual manner. The crude mixture of VII, VIII, and I was dissolved in chloroform and exhaustively extracted with $5 \%$ aqueous sodium bicarbonate. The chloroform phase was dried, filtered, and the desired product recovered from it by fractional precipitation with Skellysolve B. The yield was $777 \mathrm{mg}$ of white solid, $\mathrm{mp} 246 \sim 248^{\circ} \mathrm{C}$ (decomp). Its structure was confirmed by infrared and $\mathrm{nmr}$ spectra, and by its elemental analyses (See Table 3 ).

3-(3-Methoxy-2-nitrobenzamido)-4-hydroxy-8-methyl-7-[3-O-(5-methyl-2-pyrrolylcarbonyl)noviosyloxy]coumarin. (Compound 46). Method D.

3-Methoxy-2-nitrobenzoic acid, $24.68 \mathrm{~g}$ (125.2 millimoles) was dissolved in $200 \mathrm{ml}$ of tetrahydrofuran, $21.00 \mathrm{ml}(15.20 \mathrm{~g}, 150.2$ millimoles $)$ of triethylamine was added, and the mixture was cooled in an ice bath to $0 \sim 5^{\circ} \mathrm{C}$. Ethyl chloroformate, $13.11 \mathrm{ml}(15.0 \mathrm{~g}, 138$ millimoles), was added and the mixture was stirred at $0^{\circ} \mathrm{C}$ for 45 minutes. The resulting solution of mixed anhydride was carefully added to a pre-cooled solution of $20.00 \mathrm{~g}$ (15.65 millimoles) of ditetrahydropyranyl coumermycin $A_{1}$ (II) in $400 \mathrm{ml}$ of tetrahydrofuran containing $4.40 \mathrm{ml}$ (31.3 millimoles) of triethylamine. The reaction mixture was stirred at $0 \sim 5^{\circ} \mathrm{C}$ for 1.5 hours, then at room temperature for 20 hours. The insoluble triethylamine hydrochloride was filtered off and the filtrate was concentrated to ca. $200 \mathrm{ml}$ in vacuo. The deep yellow concentrate was poured into 2.0 liters of ice-cold Skellysolve B. 
The yellow precipitate was separated and dried, $47.8 \mathrm{~g}$. The tetrahydropyranyl groups were removed with p-toluenesulfonic acid catalysis in methanol-acetone as previously described to yield the coumermycin bis imide (confirmed by absence of amide $\mathrm{NH}$ bands in the infrared spectrum). The bis imide was cleaved to amide by warming in pyridine at $55^{\circ} \mathrm{C}$ for 48 hours. The pyridine was removed in vacuo and the residue was taken up in ca. $500 \mathrm{ml}$ of ethyl acetate and extracted 7 times with $150 \mathrm{ml}$ portions of $5 \%$ aqueous sodium bicarbonate. The solvent phase was dried and filtered, and the product was recovered by fractional precipitation with Skellysolve B. The vield of purified title compound was $465 \mathrm{mg}$.

3-(4-Aminobenzamido)-4-hydroxy-8-methyl-7-[3-O-(5-methyl-2-pyrrolylcarbonyl)noviosyloxy]coumarin. (Compound 12). Method E.

A $6.37-\mathrm{g}$ sample $(0.0100$ mole) of 3-(4-nitrobenzamido)-4-hydroxy-8-methyl-7-[3-O-(5methyl-2-pyrrolylcarbonyl)noviosyloxy]coumarin was dissolved in $200 \mathrm{ml}$ of absolute ethanol and $637 \mathrm{mg}$ of $5 \% \mathrm{Pd}$ on carbon was added. Reduction was carried out in a $500 \mathrm{ml}$ Parr hydrogenator at an initial pressure of 50 psi for 17.7 hours. The catalyst was filtered off and washed with a small amount of ethanol. The combined filtrates were reduced to dryness in vacuo and the gummy solid was dissolved in $100 \mathrm{ml}$ of ethyl acetate. The solution was heated to reflux and $10 \mathrm{ml}$ of Skellysolve B was added. Cooling produced a dark brown precipitate which was filtered off. In this manner an additional seven fractions were obtained, the last of which was a light cream color and weighed $747 \mathrm{mg}$. Analysis showed it to be pure (see Table 3) and its nmr spectrum was entirely consistent with the expected structure. The infrared spectrum showed the absence of the $1350 \mathrm{~cm}^{-1}$ band for $-\mathrm{NO}_{2}$, which was prominent in the starting material.

3-(3-Benzamido-4-hydroxybenzamido)-4-hydroxy-8-methyl-7-[3-O-(5-methyl-2-pyrrolylcarbonyl) noviosyloxy]coumarin. (Compound 45). Method F.

A solution of $150 \mathrm{mg}$ (0.241 millimole) of 3-(3-amino-4-hydroxybenzamido)-4-hydroxy8-methyl-7-[3-O-(5-methyl-2-pyrrolylcarbonyl)noviosyloxy]coumarin (Compound 42) in 5.0 $\mathrm{ml}$ of pyridine was prepared by stirring at $25^{\circ} \mathrm{C}$. Benzoic anhydride, $65.4 \mathrm{mg}(0.289$ millimole), was added and the solution was stirred at $25^{\circ} \mathrm{C}$ for 17 hours. It was poured into $250 \mathrm{ml}$ of ice water and acidified to $\mathrm{pH} 1.5$ with $6 \mathrm{~N}$ hydrochloric acid. The suspension was stirred for 30 minutes, filtered, washed and dried to give $168 \mathrm{mg}$ of crude product. It was dissolved in ethyl acetate, extracted thoroughly with $5 \%$ aqueous sodium bicarbonate solution, and the solvent phase was dried over sodium sulfate. The product was precipitated by the addition of Skellysolve B, $75 \mathrm{mg}, \mathrm{mp} 147^{\circ} \mathrm{C}$ (decomp.).

2-Picolinoyl chloride

2-Picolinic acid, $12.3 \mathrm{~g}(0.100$ mole) was heated at reflux with $50 \mathrm{ml}$ of thionyl chloride for 2.0 hours, and the excess reagent was removed under vacuum. The residue was flashed to dryness several times with Skellysolve B in order to remove excess thionyl chloride. The resulting solid was crystallized from a small volume of dry benzene, giving the title compound as dark green crystals. The infrared spectrum showed it to be ca. $95 \%$ pure acid chloride.

3-(2-Picolinamido)-4-hydroxy-8-methyl-7-[3-O-(5-methyl-2-pyrrolylcarbonyl) noviosyloxy]coumarin. (Compound 55). Method G.

A solution of $12.8 \mathrm{~g}$ (10.0 millimoles) of ditetrahydropyranyl coumermycin $A_{1}$ (II) in $320 \mathrm{ml}$ of tetrahydrofuran containing $12.8 \mathrm{ml}$ of dry pyridine was treated successively with $5.60 \mathrm{~g}$ ( 40.0 millimoles) of picolinoyl chloride and $0.36 \mathrm{ml}\left(20\right.$ millimoles) of water at $25^{\circ} \mathrm{C}$. The solution at this time was bright blue. It was stirred at $25^{\circ} \mathrm{C}$ for 6.0 hours with no further color change, concentrated to ca. $100 \mathrm{ml}$ in vacuo, and poured into 1.0 liter of ice water. The $\mathrm{pH}$ was adjusted to 4.7 with $6 \mathrm{~N}$ hydrochloric acid, and the precipitated solid was collected and dried, $10.8 \mathrm{~g}$. Imide cleavage of the intermediate $\mathrm{IV}(\mathrm{R}=2 \mathrm{Py})$ was done by dissolving the $10.8 \mathrm{~g}$ in $1080 \mathrm{ml}$ of pyridine and heating at $50^{\circ} \mathrm{C}$ for 19 hours. 
The solution was cooled to $25^{\circ} \mathrm{C}$ and concentrated to $c a .350 \mathrm{ml}$ in vacuo. It was poured into 3.5 liters of ice water and the $\mathrm{pH}$ was adjusted to 5.0 with $6 \mathrm{~N}$ hydrochloric acid. The resulting solid was filtered off and dried, giving $10.6 \mathrm{~g}$ of intermediate $\mathrm{V}(\mathrm{R}=2-\mathrm{Py})$. The tetrahydropyranyl group was removed by stirring the $10.6 \mathrm{~g}$ of intermediate in a mixture of $70 \mathrm{ml}$ of acetone, $280 \mathrm{ml}$ of methanol, and $2.7 \mathrm{~g}$ of $p$-toluenesulfonic acid monohydrate at $25^{\circ} \mathrm{C}$ for 1.0 hour. The solution was concentrated in vacuo to a syrup and poured into $c a .200 \mathrm{ml}$ of water. Fractional precipitation of the resulting solid from ether with $n$-hexane yielded the product as the second and third precipitates, combined weight $380 \mathrm{mg}$. The infrared and $\mathrm{nmr}$ spectra of this product were in full agreement with the structure of the title compound.

3-Dibenzoylamino-4-hydroxy-8-methyl-7-[2-O-tetrahydropy ranyl-3-O-(5-methyl-2pyrrolylcarbonyl)noviosyloxy]coumarin.

Ditetrahydropyranyl coumermycin $A_{1}$ (II), $1.00 \mathrm{~g}$ (0.734 millimole) was dissolved in $20 \mathrm{ml}$ of freshly distilled pyridine. During a five-minute period, $0.85 \mathrm{ml}(0.736$ millimole) of benzoyl chloride was added, the temperature being maintained at $25^{\circ} \mathrm{C}$, and the solution was stirred at $25^{\circ} \mathrm{C}$ for 24 hours, during which time it turned a deep orange-brown. It was poured into $200 \mathrm{ml}$ of vigorously stirred ice water and the $\mathrm{pH}$ was adjusted to 1.5 with $6 \mathrm{~N}$ hydrochloric acid $(40 \mathrm{ml})$. The mixture was stirred for 1 hour, the solid was filtered off, washed with water, and dried in vacuo. The crude product, $1.47 \mathrm{~g}$, was crystallized from chloroform and Skellysolve B to give $1.20 \mathrm{~g}$ of the title compound, mp $128 \sim 130^{\circ} \mathrm{C}$ (decomp.). The infrared spectrum ( $\mathrm{KBr}$ pellet) showed strong imide carbonyl bands at 1740 and $1710 \mathrm{~cm}^{-1}$, and the absence of amide $\mathrm{NH}$ near $1540^{-1}$. Integration of the $\mathrm{nmr}$ spectrum revealed the expected ratio of aromatic to aliphatic protons.

Analysis. Calculated for $\mathrm{C}_{43} \mathrm{H}_{44} \mathrm{O}_{12} \mathrm{~N}_{3}$ : C 66.14, H 5.67, N 3.59.

Found: $\mathrm{C} 66.00, \mathrm{H} 5.09, \mathrm{~N} 3.50$.

3--Dibenzoylamino-4-hydroxy-8-methy]-7-[3-O-(5-methyl-2-pyrrolylcarbonyl $)$ noviosyloxy] coumarin.

The tetrahydropyranyl group was cleaved from the preceding compound by toluenesulfonic acid-catalyzed exchange with methanol in a reaction analogous to that used in the preparation of compound 1, Method A. The product $(0.60 \mathrm{~g})$ had a decomposition point of $173 \sim 175^{\circ} \mathrm{C}$ and was devoid of antibacterial activity. Its infrared and $\mathrm{nmr}$ spectra were entirely consistent with the named structure.

Analysis. Calculated for $\mathrm{C}_{38} \mathrm{H}_{36} \mathrm{O}_{11} \mathrm{~N}_{2}$ : C 65.51, H 5.21, N 4.02 .

Found: C 65.64, H 5.35, N 3.93 .

The $\mathrm{N}$-dibenzoylimide was readily cleaved in warm pyridine $\left(50^{\circ} \mathrm{C}, 24\right.$ hours $)$ to give a high yield of 3-benzamido-4-hydroxy-8-methyl-7-[3-O-(5-methyl-2-pyrrolylcarbonyl) noviosyloxy]-coumarin, compound 1 , which was identical in all respects with previously characterized material.

3-Dibenzoylamino-4-hydroxy-8-methyl-7--[2-O-benzoyl-3-O-(5-methyl-2-pyrrolylcarbonyl) noviosyloxy]coumarin.

When the procedure described above for 3-dibenzoylamino-4-hydroxy-8-methyl-7-[2O-tetrahydropyranyl-3-O-(5-methyl-2-pyrrolylcarbonyl)-noviosyloxy]coumarin was carried out on coumermycin $A_{1}$, I, the title compound was obtained, mp $175 \sim 180^{\circ} \mathrm{C}$ (decomp.). The infrared and nmr spectra were entirely consistent with the named structure, and the compound had no antibacterial activity.

Analysis. Calculated for $\mathrm{C}_{45} \mathrm{H}_{40} \mathrm{O}_{12} \mathrm{~N}_{2}$ : C $67.49, \mathrm{H} 5.04, \mathrm{~N} 3.50$.

Found: C 67.36, H 5.07, N 3.52 .

Dibenzoyl coumermycin $A_{1}$ bis imide, via intermediate IV $\left(\mathrm{R}=\mathrm{C}_{6} \mathrm{H}_{5}\right)$.

Ditetrahydropyranyl coumermycin $\mathrm{A}_{1}$ (II), $5.00 \mathrm{~g}$ (3.91 millimoles) was dissolved in $100 \mathrm{ml}$ of freshly distilled pyridine and the solution was cooled to $-5^{\circ}$ to $-10^{\circ} \mathrm{C}$. Benzoyl chloride, $4.60 \mathrm{ml}$ (39.1 millimoles) was carefully added at a rate such that no 
temperature rise was observed, and the reaction mixture was stirred under a nitrogen atmosphere for 6 hours at $-5^{\circ} \mathrm{C}$. The pyridine hydrochloride which precipitated was removed by filtration and the filtrate was poured into 1.0 liter of ice water. The crude product which precipitated was filtered off, washed with ice water and dried in vacuo. It was thoroughly washed with carbon tetrachloride and again dried, yield $6.6 \mathrm{~g}$. At this stage, the infrared spectrum showed the absence of amide $\mathrm{NH}$ near $1540 \mathrm{~cm}^{-1}$ and had strong carbonyl bands at 1680 $1750 \mathrm{~cm}^{-1}$, which suggested the imide structure. The presence of the central 3-methylpyrrole-2,4-dicarboxamide moiety was shown by nmr bands at $2.42 \mathrm{ppm}$ and $11.2 \mathrm{ppm}$ (in $\mathrm{CDCl}_{3}-\mathrm{DMSO}$ ) which are characteristic of the D-pyrrole methyl and $\mathrm{NH}$ groups respectively. The tetrahydropyranyl blocking groups were removed with $p$-toluenesulfonic acid catalyst in methanol-acetone, as previously described. The resulting product, $660 \mathrm{mg}$, had $\mathrm{mp} 182 \sim 185^{\circ} \mathrm{C}$ (decomp.). The bis imide formulation was fully supported by its infrared and nmr spectra.

Analysis. Calculated for $\mathrm{C}_{69} \mathrm{H}_{67} \mathrm{O}_{22} \mathrm{~N}_{5} \cdot \mathrm{H}_{2} \mathrm{O}$ : C 62.01, H 5.20, N 5.24.

Found: C $62.07, \mathrm{H} 5.34, \mathrm{~N} 5.23$.

Solvolysis of the above bis imide in pyridine at $55^{\circ} \mathrm{C}$ for 48 hours gave, after recovery of product as described in Method A, the expected compound 1. Comparison of its infrared and nmr spectra, chromatographic behavior, elemental analyses, and bioassay with previously characterized compound 1 showed them to be identical.

Compounds $16,17,18,34,35,39,40,41,48$, and 50 were all prepared via transacylation with the corresponding acetoxybenzoyl chlorides. After imide cleavage in warm pyridine and tetrahydropyranyl cleavage in methanol-acetone with $p$-toluenesulfonic acid, some of the phenolic hydroxyls in each sample remained acetylated. Although these acetoxy compounds had some bioactivity, it was always raised by complete removal of acetoxy groups as follows: The crude cleavage mixture of VII, VIII, and I was dissolved in as much liquid ammonia as required to give a clear solution (frequently ca. $10 \mathrm{ml} / \mathrm{gram}$ of sample). It was allowed to evaporate at room temperature, and the residue was dissolved in water. On adjustment of the $\mathrm{pH}$ to $c a .1 .5$ with dilute hydrochloric acid, the crude product mixture (VII, VIII, and I, now minus all acetoxy groups) precipitated. It was filtered off, dried, and the desired compounds VII were obtained in each case by fractional precipitation from ethyl acetate or chloroform solution with Skellysolve B.

3-(Thiazole-4-carboxamido)-4-hydroxy-8-methyl-7-[3-O-(5-methyl-2-pyrrolylcarbonyl) noviosyloxy]coumarin. (Compound 54).

Thiazole-4-carboxylic acid was converted to the acid chloride by brief reflux with excess thionyl chloride. The excess reagent was completely removed in vacuum, and a solution containing $12.8 \mathrm{~g}$ (10.0 millimoles) of ditetrahydropyranyl coumermycin $\mathrm{A}_{1}$ and $180 \mathrm{mg}$ of water in $200 \mathrm{ml}$ of pyridine was added to $5.88 \mathrm{~g}$ ( 40.0 millimoles) of thiazole4 -carbonyl chloride. The mixture was kept at $50^{\circ} \mathrm{C}$ for 24 hours, and the crude product was precipitated by adding the solution to 2 liters of ice water and acidifying to $\mathrm{pH} 4.5$ with dilute hydrochloric acid. It was filtered off, dried, and dissolved in a mixture of $200 \mathrm{ml}$ of acetone and $30 \mathrm{ml}$ of methanol containing $600 \mathrm{mg}$ of $p$-toluenesulfonic acid monohydrate. It was agitated for 24 hours, then concentrated to a small volume and diluted with water. The resulting precipitate was dissolved in ethyl acetate, exhaustively extracted with $5 \%$ aqueous sodium bicarbonate, and dried over sodium sulfate. Partially purified product, $5 \mathrm{~g}$, was obained by fractional precipitation with Skellysolve B. Three grams of this product was dissolved in ca. $15 \mathrm{ml}$ of $1: 1$ acetone : methanol and fractionated on a $2.5 \times 35 \mathrm{~cm}$ column of Sephadex LH20, which was prepared and developed with the same solvent. The peak fractions, as determined by maximal ultraviolet absorption at $365 \mathrm{~m} \mu$ in ethyl acetate and by maximal antibiotic assay, were combined, flashed dry, and the residue crystallized from ethyl acetate and Skellysolve B. The infiared and nmr spectra substantiated the structure. 
Analysis. Calculated for $\mathrm{C}_{28} \mathrm{H}_{29} \mathrm{O}_{10} \mathrm{~N}_{5} \mathrm{~S}: \mathrm{N} 7.01$, S 5.35 .

Found: N 7.30, S 5.12.

\section{Literature Cited}

1) BeLL, F.: The migration of acyl groups in 0 -aminophenols. II. J. Chem. Soc. $1931: 2962 \sim 2967$, 1931.

2) Cunningham, B.A. \& G. L. Schmir: Hydroxyl group participation in amide hydrolysis. The infuence of catalysts on the partitioning of a tetrahedral intermediate. J. Am. Chem. Soc. $89:$ : 17 $222,1967$.

3) Hinman, J. W.; H. Hoemsema, E. L. Caron \& W. G. JaCkson: The partial structure of novobiocin (streptonivicin). II. J. Am. Chem. Soc. $78: 1072 \sim 1074,1956$.

4) Kakwaguchi, H.; M. OKanishi \& T. Miyaki: Coumermycin and salts thereof. U.S. Pat. 3,201,386, Aug. 17, 1965.

5) Kawaguchi, H.; H. Tsukiura, M. Okanishi, T. Miyakt, T. Ohmori, K. Fujisawa \& H. Koshiyama: Studies on coumermycin, a new antibiotic. I. Production, isolation and characterization of coumermycin $A_{1}$. J. Antibiotics, Ser. A $18: 1 \sim 10,1965$. 